\title{
High Accuracy Detection and Tracking of Objects
}

\author{
Mr. V. M. Vijay Kannan, \\ Faculty of Computer Science \& Engg Dept, Erode Sengunthar Engineering College, Erode, Tamilnadu, India.
}

\begin{abstract}
One of the critical tasks in Computer Vision is Detection and Tracking of objects. But still now, the issues related to this are developing. For the automatic detection of moving objects, some of the monitoring systems cannot able to find the difference, when the difference of brightness between the background and the moving objects is small. The costs are very high in these systems. Most of the previous methods, only concentrated on detecting rough area of targets. The accurate moving target detection cannot be achieved. It makes the result to be shown with noise and heals and the computation time is also increased. Many systems are unable to solve critical solutions such as Partial Occlusions and Cross Targets. In my proposed system the Soft Computing techniques can handle objectives and arbitrary constraints with a high degree of simplicity, we use one of the Evolutionary Approach of Soft Computing Technique in this paper. We demonstrate the object detection and tracking methods separately. Initially, a visual object detection approach is presented for minimizing the computation time, in order to achieve high detection accuracy. In this approach, three key contributions are described.
\end{abstract}

Key terms: Video surveillance, Soft computing, object detection, tracking.

\section{Introduction}

Video surveillance is an important research area in the business-related sector. Technology has reached a stage where mounting cameras to capture video imagery is cheap, but finding available human resources to sit and watch that imagery is expensive. Surveillance cameras are already prevalent in commercial establishments, with camera output being recorded to taps that are either rewritten periodically or stored in video archives [1]. One of the goals in video surveillance is to detect and track all the relevant moving objects in the scene, and to generate exactly one track per object. This may involve detecting the moving objects, tracking them while they are visible, and re-acquiring the objects once they emerge from an occlusion to maintain identity. This is a very difficult problem, even more so when the sensor is moving, as in aerial surveillance scenarios [2].

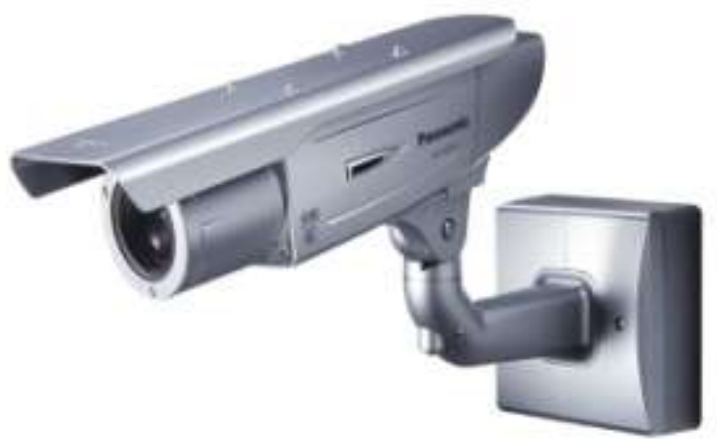

The Motion analysis is a basis of all intelligent video surveillance technologies. In particular, it provides the fundamentals for automatic detection and tracking of moving objects and automatic detection of new or disappeared objects of observed scene. It is the well-studied area of computer vision including many different techniques [6]. Object detection and tracking focuses on detecting the position of a moving object in a video sequence. It is the first step accomplished by a event recognition system that extracts semantic content from video [11]. The purpose of detecting moving objects in video stream is to be able to track the objects over time and derive a set of properties from their trajectory such as their behaviours [9].

A good detection measure should capture the performance in terms of both overall detection (number of objects detected, missed detects and false alarms) and goodness of detection for the detected objects, i.e., spatial accuracy (how much of the ground truth is detected) and spatial fragmentation (object splits and object merges) [6]. In general, there are two types of approaches in Motion detection : (i) the region-based approach and (ii) the boundary-based approach [4]. Another approaches to object detection for surveillance, including feature-based object detection, template-based object detection and background subtraction or inter-frame difference-based detection [7]. 
The goal of the tracking stage is to use the observations to generate the tracks that correspond to the moving objects. Some of the issues that make this a challenging problem include missed observations due to poor video quality, noisy observations due to changes in illumination, occlusions, objects that are moving in close proximity to each other, etc [10]. frequently used approaches for tracking are token-based, when a geometric description of the object is available, or intensity-based (optical flow, correlation...). These techniques are not that much appropriate for blob tracking, since a reliable geometric description of the blobs cannot be inferred. On the other hand, the intensity-based techniques are ignore the geometric description of the blob [9]. The tracking-based methods encounter the problems of merging, splitting, entering, leaving, occlusion, and correspondence.

These problems are not easy to solve in many cases. And it is difficult to track all the objects precisely in crowded situations [8]. To track from a moving camera, we need to describe the motion of moving objects in common coordinates. The mosaic space, which is derived from the image coordinates of one frame, is usually selected as the tracking coordinates. Without further refinement, accumulated errors are inevitable if fixed coordinates are selected. More importantly, an object motion in image coordinates is not physically meaningful [2].

A typical surveillance application consists of three building blocks, responsible of: moving object detection, object tracking and higher level motion analysis. Applications include car and pedestrian traffic monitoring, human activity surveillance for unusual activity detection, people counting etc., [3]. DETECTION of moving objects in the video streams is the first important step of information extraction in many computer vision applications, including video surveillance, people tracking, traffic monitoring and semantic annotation of videos. In these applications, tracking of correct objects in the scene calls robust for a reliable and effective moving object detection that should be characterized by some important features: high precision, with the two meanings of accuracy in shape detection and reactivity to changes in time; flexibility in different scenarios (indoor, outdoor) or different light conditions; and efficiency, in order for detection to be provided in real-time [5]. Detecting and tracking the moving objects are commonly used as low-level tasks of computer vision applications, such as video surveillance, robotics, authentication systems, user interfaces by gestures, and a prestage of MPEG4 image compression. Software development of low-level tasks is especially important because it influences the performance of all higher levels of various applications [4].

\section{Analysing Various Video Surveillance Techniques}

Video surveillance is defined as the task of analyzing video sequences to detect abnormal and unusual activities. Moving object detection is the basic step for further analysis of video. Commonly all tracking method requires an object detection mechanism either in every frame or when the object first appears in the video. Object tracking is the process, which is used to track the object over the time by locating its position in every frame of the video in surveillance system. A brief review of some recent researches is presented here.

Adnan Khashman [12] has presented an automatic moving object detection, extraction, and recognition system (aMODERs). The system received still images captured every two seconds from a surveillance camera, which monitors a restricted zone, such as international border crossings, buffer zone in conflict areas, or any monitored area where movement across that area is to be detected. The potential objects which have been considered in his work were classified into three groups: human (female, male), animals (goat), and vehicles (motorbike, car, loader, jeep).The use of (aMODERs) in a real life application was not limited to those objects, because the neural network classifier could be quickly and easily trained to recognize as many different objects as required. The system provided solutions to the problem of monitoring secured areas; namely, the detection of movement across the area, the extraction of the moving object, and the recognition of the object.

Neelam Patel [13] has formed a series of algorithm, to track the feature of motion detection under surveillance system. A pixel variant played a vital role in detection of moving frame of a particular clip. If there was a little bit motion in a file then it was detected very easily by tracking pixel variance. The algorithm detected the zero variation only when there was no motion in a frame clip. It was simple and easier for motion detection in the frames of moving object having Avi file. There were different levels at which tracking can be performed. At the highest level, the whole body was tracked without paying attention to the details of the posture and limbs. At a lower level, the posture and limbs were tracked. At an even lower level, one or two parts of the body (such as hands) were tracked. The finest level would be tracking the fingers of a hand or facial features. The Scope of his paper was dealt with the highest level of tracking (tracking the Whole body as one unit). His paper answered the crucial question regarding the tracking of an object and suggested the requirement of surveillances in the terms of security and in high tech world. 
Video surveillance is gaining its importance in almost every field of day to day life. Surveillance is being done not only in military areas or airports but also in offices, schools, shopping areas, old age home and many more areas. Garima Sharma [14] has presented a general review on the overall process of a surveillance system used in the present time. The processing framework of the video surveillance system included the following stages: moving object detection, object segmentation, representation, classification, tracking of objects, activity recognition and prediction. The video surveillance system could be classified in a number of areas considering the environment in which they operate: indoor, outdoor or airborne.

Amir Aliabadian et al. [15] have proposed and tested a modified object-tracking algorithm that uses the flexible Metric Distance Transform kernel and multiple features for the Mean shift procedure. The Faithful target separation based on RGB joint pdf of the target region and that of a neighbourhood surrounding the object was obtained. The non-linear log-likelihood function mapped the multimodal object/background distribution as positive values for colors associated with foreground, while negative values were marked for background. This replaced the more usual Epanechnikov kernel (E-kernel), improving target representation and localization without increasing the processing time, minimizing the similarity measure using the Bhattacharya coefficient.

Pande R.P. et al. [16] have proposed a new method to detect moving object based on background subtraction. First of all, they have established a reliable background updating model based on statistical and used a dynamic optimization threshold method to obtain a more complete moving object. And then, morphological filtering was introduced to eliminate the noise and solve the background disturbance problem. At last, contour projection analysis was combined with the shape analysis to remove the effect of shadow; the moving human bodies were accurately and reliably detected. In a single static camera condition, they combined dynamic background modeling with dynamic threshold. Selection method based on the background subtraction, and up-date background on the basis of accurate detection of object, the method was effective to enhance the effect of moving object detection.

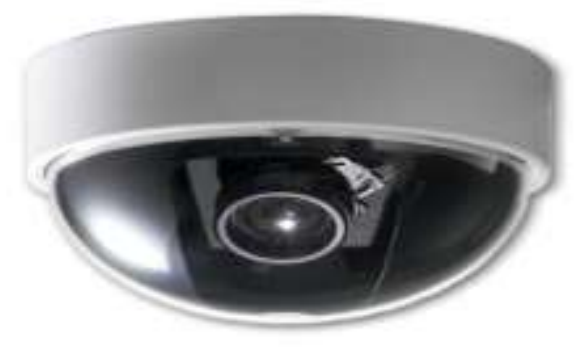

Kinjal A Joshi, and Darshak G. Thakore [17] have presented a survey of various techniques related to video surveillance system improving the security. The goal of their paper was to review of various moving object detection and object tracking methods. They have focused on detection of moving objects in video surveillance system then tracking the detected objects in the scene. Moving Object detection was first low level important task for any video surveillance application. Detection of moving object was a challenging task. Tracking was required in higher level applications that require the location and shape of object in every frame. They have described background subtraction with alpha, temporal differencing, statistical methods. They gave valuable insight into the important research topic and encouraged the new research in the area of moving object detection as well as in the field of computer vision. Their research on object tracking could be classified as point tracking, kernel tracking and contour tracking according to the representation method of a target object.

Chih-Hsien Hsia, and Jen-Shiun Chiang [18] have presented a new approach, direct LL-mask band scheme (DLLBS), for the detection and tracking of moving objects using a low resolution image. Moving object detection was an important basic task for intelligent video surveillance systems, because it provided a focus of attention for post-processing. However, the successful detection of moving objects in a real environment was a difficult task, due to noise cause by fake motion, such as the motion of leaves in trees. Many methods have been developed in constrained environments, for the detection and tracking of moving objects. The DLLBS method could effectively reduce the noise, with low computing cost, in both indoor and outdoor environments. For circumstances where occlusions occur, they have proposed a new approach, characteristic point recognition (CPR). Together with DLLBS and CPR, the problems associated with occlusions were alleviated.

Ritika, and Gianetan Singh Sekhon [19] have discussed an efficient and effective approach for identifying and tracking of moving object from a video. A video was captured by stationary camera. Moving object tracking and detection from video sequences has applications in several areas such as automatic video surveillance, motion-based recognition, video indexing, human-computer interaction, traffic monitoring, and vehicle navigation. They have presented a computer vision-based approach for object tracking and detection. A method was proposed to detect and track moving object through video even if 
background was changed at any instant and capable of plotting a 3D graph mesh based on the moving object in between any number of frames per second. They have used consecutive frame analysis technique to detect background changing criteria and use morphological filtering for image enhancement. Finally, they got the co-ordinates for the moving object and these co-ordinates are imported to any other 3D software's like MAYA etc., to analyze or edit the results calculated by the algorithm.

Jun Zhang et al. [20] have proposed a two-step accurate moving target detection method. Their method obtained a rough target area using a color background subtraction method with a feedback to background estimation, followed by a modified SUSAN method to estimate the accurate target edge. In the first step, they used the color background subtraction method and morphology method to detect the rough area of target. In the second step, a modified SUSAN method was proposed to detect the accurate edge of target in the rough area. In the case of noisy video frames, some pixels are recognized as background, holes appear in the estimation results. Holes need to be filled to get the whole target area. After filling the holes, we get the accurate area of moving target. The background area is returned to background estimation as a feedback to update the background. The modified SUSAN method used the grads magnitudes to achieve an adaptive threshold and the gray barycenter criterion for denoising.

The surveillance detection related events from video input is a sophisticated technology in real time security related applications. Many current and Existing solutions to this problem are simply slight variations on frame differencing concept. This proves to be difficult to configure and operate effectively. Sahithi, and Venkat Mutyalu [21] have presented a new approach based on extracting and classifying the background contents of each video frame using a CAM equipped with a standard framegrabber. The action of objects classified as people was further categorized into a series of events such as person leaving, entering, deposits object, and so forth. A background model was used to obtain candidate surveillance objects from input video. The focus of their research was to detect and predict anomalous behaviour of individuals in an environment using a camera as a way to capture the information about the environment.

\section{Proposed Methodology}

Video Surveillance has been an active recent topic in computer vision, which attempts to detect and track the objects from image sequences. In order to reduce the computation time, while detecting objects with a high detecting accuracy and to track the object accurately, we need to use Soft Computing techniques. Because of the fact that the Soft Computing techniques can handle objectives and arbitrary constraints with a high degree of simplicity, we use one of the Evolutionary Approach of Soft Computing Technique in this paper. We demonstrate the object detection and tracking methods separately. Initially, a visual object detection approach is presented for minimizing the computation time, in order to achieve high detection accuracy. In this approach, three key contributions are described.

First contribution is to represent new image features using "Integral Image". The second is to the selection of image features based on "AdaBoost learning algorithm", which is used to boost the classification performance of a simple learning algorithm. The third one is a technique to combine the classifiers in a "cascade" that reduces the computation time by allowing background regions of images to be discarded quickly. In overall, the detection process is that of a degenerate decision tree. After finishing the detection process, we present a novel genetic algorithm based on region growing and matching scheme, for the purpose of object tracking. The aim of this algorithm is to track the objects accurately. Region growing scheme starts with selecting a seed region and developing these regions with other neighboring regions based on the output of genetic algorithm based region matching. From the proposed methodology, we can prove that both of our object detection and tracking methods using such Soft Computing techniques achieve high detection accuracy with low computation time and also achieve accurate tracking of objects.

\section{Conclusion}

One of the critical tasks in Computer Vision is Detection and Tracking of objects. But still now, the issues related to this are developing. For the automatic detection of moving objects, some of the monitoring systems cannot able to find the difference, when the difference of brightness between the background and the moving objects is small. The costs are very high in these systems. Most of the previous methods, only concentrated on detecting rough area of targets. The accurate moving target detection cannot be achieved. It makes the result to be shown with noise and heals and the computation time is also increased. Many systems are unable to solve critical solutions such as Partial Occlusions and Cross Targets. Even if the partial occlusions happened, because of other objects or the crossing of other moving objects, the systems cannot have the ability to keep tracking its target. The object tracking can be complex, due to camera motion, loss of information caused by the projection of the 3D world on a 2D image, variations of target scale, partial occlusions, real-time processing requirements, clutter, and so on. Therefore it is difficult to make the efficient tracker and also we cannot track the object accurately. 


\section{References}

[1] Sherin M. Youssef, Meer A. Hamza, and Arige F. Fayed, "Detection and Tracking of Multiple Moving Objects with Occlusion in Smart Video Surveillance Systems", In Proceedings of IEEE Conference of Intelligent Systems, pp. 120-125, 2010.

[2] Yuping Lin, Qian Yu, and Gérard Medioni, "Efficient detection and tracking of moving objects in geo-coordinates", Machine Vision and Applications, Vol. 22, pp. 505-520, 2011.

[3] Codrut Ianasi, Vasile Gui, Corneliu I. Toma, and Dan Pescaru, "A Fast Algorithm for Background Tracking in Video Surveillance, Using Nonparametric Kernel Density Estimation", Electronics and Energetics, Vol. 18, No. 1, pp. 127-144, 2005.

[4] Masayuki Yokoyama, and , "A Contour-Based Moving Object Detection and Tracking", In proceedings of Visual Surveillance and Performance Evaluation of Tracking and Surveillance, 2005.

[5] Rita Cucchiara, Costantino Grana, Massimo Piccardi, and Andrea Prati, "Detecting Moving Objects, Ghosts and Shadows in Video Streams", IEEE Transactions on Pattern Analysis and Machine Intelligence, Vol. 25, pp. 1337-1342, October 2003.

[6] Vasant Manohar, Padmanabhan Soundararajan, HarishRaju, Dmitry Goldgof, Rangachar Kasturi, and John Garofolo, "Performance Evaluation of Object Detection and Tracking in Video", In Proceedings of 7th Asian Conference on Computer Vision, Hyderabad, pp. 151-161, 2006.

[7] Kaiqi Huanga, Liangsheng Wanga, Tieniu Tana, and Steve Maybank, "A real-time object detecting and tracking system for outdoor night surveillance", ELSEVIER Pattern Recognition, Vol. 41, pp. 432-444, 2008.

[8] Thi Thi Zin, Pyke Tin, Takashi Toriu and Hiromitsu Hama, "A Probability-based Model for Detecting Abandoned Objects in Video Surveillance Systems", In Proceedings of the World Congress on Engineering 2012, London, U.K., Vol 2, 2012.

[9] Isaac Cohen, and Gerard Medioni, "Detecting and Tracking Moving Objects for Video Surveillance", In IEEE Proceedings of Computer Vision and Pattern Recognition, pp. 2319-2325, 1999.

[10] C. Kamath, A. Gezahegne, S. Newsam, and G. M.Roberts ,"Salient Points for Tracking Moving Objects in Video", Image and Video Communications and Processing, 2005.

[11] Boris Vishnyakov, Yury Vizilter, and Vladimir Knyaz, "Spectrum-Based Object Detection And Tracking Technique For Digital Video Surveillance", International Archives of the Photogrammetry, Remote Sensing and Spatial Information Sciences, pp. 579583,2012 .

[12] Adnan Khashman, "Automatic Detection, Extraction and Recognition of Moving Objects", International Journal of Systems Applications, Engineering and Development, Vol. 2, pp. 43-51, 2008.

[13] Neelam Patel, "Motion Detection based on Multi Frame Video under Surveillance System", International Journal of Emerging Technology and Advanced Engineering, Vol. 2, pp. 124-129, January 2012.

[14] Garima Sharma, "Video Surveillance System: A Review", International Journal of Research in Engineering and Applied Sciences, Vol. 2, pp. 75-85, February 2012.

[15] Amir Aliabadian, Esmaeil Akbarpour, and Mohammad Yosefi, "Kernel Based Approach toward Automatic object Detection and Tracking in Surveillance Systems", International Journal of Soft Computing and Engineering, Vol. 2, pp. 82-87, March 2012.

[16] Pande R.P., Mishra N.D., Gulhane S., and Joshi A., "Detection of Moving Object With the help of Motion Detection Alarm System In Video Survelliance", Journal of Signal and Image Processing, Vol. 3, pp. 118-121, 2012.

[17] Kinjal A Joshi, and Darshak G. Thakore, "A Survey on Moving Object Detection and Tracking in Video Surveillance System", International Journal of Soft Computing and Engineering, Vol. 2, pp. 44-48, July 2012.

[18] Chih-Hsien Hsia, and Jen-Shiun Chiang, "Real-Time multiple moving objects detection and tracking with direct LL-Mask Band Scheme", International Journal of InnovativeComputing, Information and Control, Vol. 8, No. 7(A), pp. 4451-4468, July 2012.

[19] Ritika, and Gianetan Singh Sekhon, "Path Estimation and Motion Detection of Moving Object in Videos", IOSR Journal of Computer Engineering, Vol. 2, pp. 01-04, July-Aug. 2012.

[20] Jun Zhang, Shukui Xu, Kuihua Huang, and Tingjin Luo, "Accurate Moving Target Detection Based on Background Subtraction and SUSAN", International Journal of Computer and Electrical Engineering, Vol. 4, No. 4, pp. 436-439, August 2012.

[21] Sahithi, and Venkat Mutyalu, "Remote Based Intelligent Video Surveillance System", International Journal of Computer Trends and Technology, vol. 3, pp. 527-530, 2012. 